\section{PerFRACTION ${ }^{T M}$}

3D Pre-Treatment QA and In-Vivo Monitoring

- Fraction $0^{\mathrm{TM}}$ Pre-treatment phantom-free QA with the independence and peace of mind of EPID measurements

- Fraction $n^{\mathrm{TM}}$ In-vivo QA for every treatment catches patient and machine errors

- See which clinical goals pass and fail for each daily fraction

- Automated DICOM file capture, analysis, and email notifications for failure

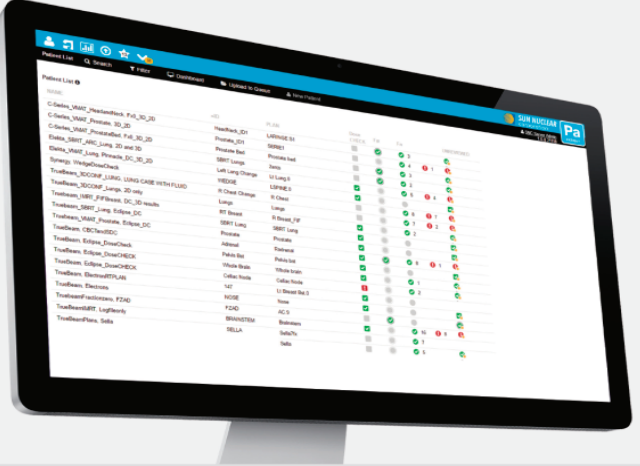

\section{Stereotactic QA}

New Solutions

\section{SRS MapCHECK}

Small Field Digital Film Replacement for Patient Specific QA \& End-to-End Testing

- Designed to insert into StereoPHAN ${ }^{m}$ and run on SNC Patient ${ }^{\text {tw }}$ software

Detector Spacing: $2.47 \mathrm{~mm}$; Detector Resolution: $0.47 \mathrm{~mm}$ diameter

- Measures field sizes as small as $5 \mathrm{~mm}$; 5 diodes in $5 \mathrm{~mm}$ cone

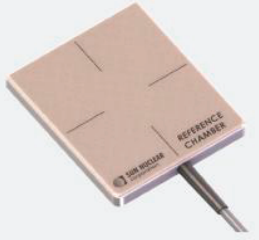

Reference Detector

An Out-of-Field Reference Detector for Water Tank Scanning

Measures LINAC head leakage, enabled by large-volume ion chamber

Used during photon beam commissioning measurements of any field size

Eliminates scanning chamber interference in small SRS fields

- Volume: 39cc; 2-meter cable with triaxial connector
DoseCHECK ${ }^{\mathrm{TM}}$

Independent Secondary 3D Dose Calculations

- Automated, TPS grade dose volume generation

- Efficient dose-to-dose evaluation

- Seamless integration with the PerFRACTION ${ }^{\mathrm{TM}}$ solution for patient QA

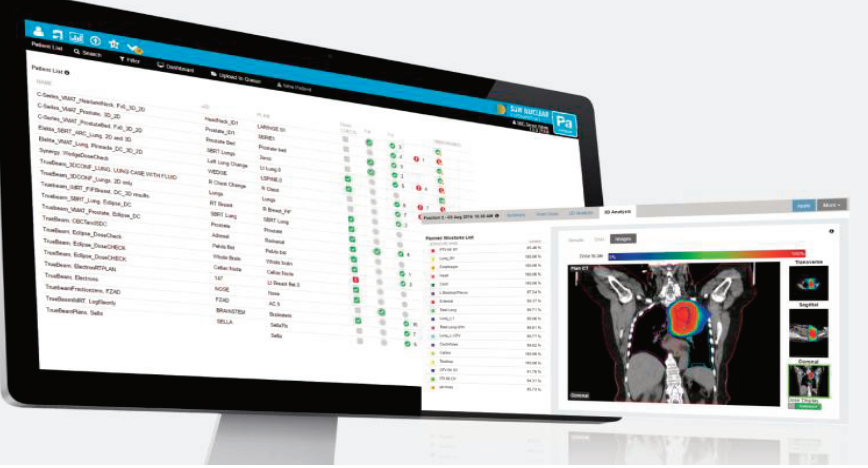

SNC Machine ${ }^{\mathrm{TM}}$

TG-142/VMAT Imaging and Mechanical QA

- Automated workflow captures and analyzes files - no further work required

- Supports most common mechanical and imaging phantoms

- Trend any test parameter against any other test parameter for any number of machines

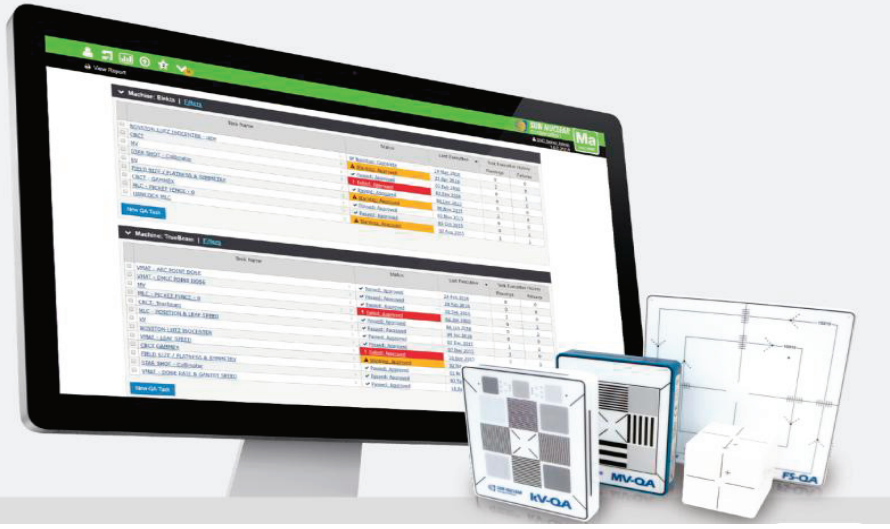




\title{
Dosimetric Evaluation of Low-Dose Spillage Volumes for Head and Neck Cancer Using Intensity-Modulated Radiation Therapy and Volumetric Modulated Arc Therapy Treatment Techniques
}

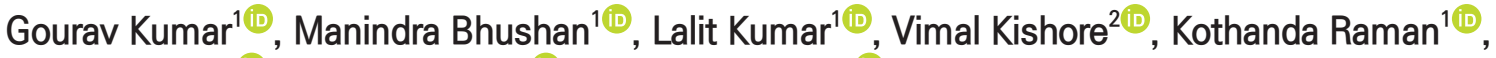 \\ Pawan Kumar $^{1(\mathbb{D})}$, Soumitra Barik ${ }^{1 \text { (i) }}$, Sandeep Purohit ${ }^{1(i)}$ \\ ${ }^{1}$ Medical Physics Division \& Department of Radiation Oncology, Rajiv Gandhi Cancer Institute and Research Centre, New Delhi, \\ ${ }^{2}$ Department of Applied Science \& Humanities, Bundelkhand Institute of Engineering \& Technology, Jhansi, India
}

Received 5 June 2021

Revised 5 August 2021

Accepted 10 August 2021

Corresponding author

Lalit Kumar

(lalitk48@gmail.com)

Tel: +91-9599329464

Fax: +91-11-27051037
Purpose: This study was designed to investigate the dosimetric difference between intensitymodulated radiation therapy (IMRT) and volumetric modulated arc therapy (VMAT) in head and neck cancer (HNC). The study primarily focuses on low-dose spillage evaluation between these two techniques.

Methods: This retrospective study involved 45 patients with HNC. The treatment plans were generated using the IMRT and VMAT techniques for all patients. Dosimetric comparisons were performed in terms of target coverage, organ-at-risk (OAR) sparing, and various parameters, including conformity index, uniformity index, homogeneity index, conformation number, low-dose volumes, and normal tissue integral dose (NTID).

Results: No significant $(P>0.05)$ difference in planning target volume coverage $\left(D_{95 \%}\right)$ was observed between IMRT and VMAT plans for supraglottic larynx, hard palate, and tongue cancers. A decrease in dose volumes ranging from $1 \mathrm{~Gy}$ to $30 \mathrm{~Gy}$ was observed for VMAT plans compared with those for IMRT plans, except for $V_{1 G y}$ and $V_{30 G y}$ for supraglottic larynx cancer and $V_{1 G y}$ for tongue cancer. Moreover, decreases $(P<0.05)$ in NTID were observed for VMAT plans compared with that for IMRT plans in supraglottic larynx (4.50\%), hard palate (12.80\%), and tongue $(7.76 \%)$ cancers. In contrast, a slight increase in monitor units for VMAT compared with those for IMRT in supraglottic larynx $(0.46 \%)$, hard palate $(2.54 \%)$, and tongue $(7.56 \%)$ cancers.

Conclusions: For advanced-stage HNC, both IMRT and VMAT offer satisfactory clinical plans. VMAT offers a conformal and homogeneous dose distribution with comparable OAR sparing and higher dose falloff outside the target volume than IMRT, which provides an edge to reduce the risk of secondary malignancies for HNC over IMRT.

Keywords: Intensity-modulated radiation therapy, Volumetric modulated arc therapy, Head and neck cancer, Low-dose spillage, Radiotherapy

\section{Introduction}

Head and neck cancers (HNCs) are the most common type of malignancy spreading worldwide. In India, among all cancer cases reported every year, $35 \%$ are HNC cases $[1,2]$. Most HNC malignancies include tumors of the tongue,

Copyright $\odot 2021$ Korean Society of Medical Physics

-This is an Open-Access article distributed under the terms of the Creative Commons Attribution Non-Commercial License (http://creativecommons.org/licenses/by$\mathrm{nc} / 4.0$ ) which permits unrestricted non-commercial use, distribution, and reproduction in any medium, provided the original work is properly cited. 
nasopharynx, oropharynx, hypopharynx, and oral cavity $[3,4]$. Among all HNCs, squamous cell carcinoma (SCC) constitutes more than $90 \%$ of the cases. The widespread use of tobacco and smoking are considered the most important factors for developing these malignancies. Due to the lack of appropriate knowledge and education, most Indian patients present with the advanced stage of the disease, therefore, making radiotherapy challenging. According to the 2018 Global Cancer Incidence, Mortality and Prevalence report, there were 834,860 new HNC cases reported every year, resulting in 431,131 deaths per year [5]. Limited options are available for treatment, such as surgery, chemotherapy, and radiotherapy for early and advanced stages of HNC.

The primary aim of radiotherapy is to deliver therapeutic doses to the target while sparing the surrounding organsat-risk (OARs) as much as possible. The introduction of intensity-modulated radiation therapy (IMRT) in HNC replaced conventional three-dimensional conformal radiotherapy (3D-CRT), resulting in better target coverage, more conformity, and better sparing of OARs [6]. Radiationinduced toxicities are low in IMRT compared with the 3DCRT technique [7]. IMRT is primarily useful for irregularly shaped target volumes to achieve a high degree of conformal dose distribution using large numbers of smaller radiation beamlets of varying intensities [8].

With the advancement in the IMRT technique, volumetric modulated arc therapy (VMAT), a rotational treatment technology, came into existence in 2007 and now widely accepted worldwide [9]. VMAT is the evolution of IMRT incorporating simultaneous variation of three parameters, namely, the gantry speed, multileaf collimator (MLC) speed, and dose rate. Because of steeper dose gradients, both IMRT and VMAT require accurate target and normal tissue delineation. Various studies in the literature have shown that VMAT delivers a more conformal dose to planning target volumes (PTVs), results in a lower dose spillage, and has a better normal tissue-sparing ability than the IMRT technique [10-14].

With the evolution of technology, dose escalation and conformity to the target has improved. However, normal tissues outside the target get irradiated unnecessarily. It has been reported in the literature that radiation exposure to normal tissues causes secondary malignancies. The risk of secondary malignancy depends on the location and amount of exposure received by the surrounding normal tissues. Diallo et al. [15] have reported that $66 \%$ of secondary malignancies occurred within the first $5 \mathrm{~cm}$ from the field boundary, $22 \%$ occurred after the first $5 \mathrm{~cm}$ from the field boundary, and $12 \%$ occurred within the target volume.

The dose distribution generated for HNC cases using IMRT and VMAT has been enormously detailed in the literature $[11,16,17]$. However, distinction in low-dose distribution generated using IMRT and VMAT takes a dip for HNC cases and should be evaluated. Low-dose exposure may contribute to secondary malignancies. Therefore, this study was designed to quantify the aforementioned difference in dose distribution generated for $\mathrm{HNC}$ cases.

This study focused on the selection of the most appropriate treatment technique between IMRT and VMAT for patients with HNC regarding the conformity index (CI), homogeneity index (HI), conformation number (CN), uniformity index (UI), and other dosimetric parameters. Few studies have reported the effects of low doses in radiotherapy planning. Therefore, this study was conducted to determine the dosimetric difference between IMRT and VMAT in terms of low-dose spillage.

\section{Materials and Methods}

\section{Patient selection, contouring, and dose prescription}

This retrospective study involved 45 patients with stage II and IIIB HNC with a mean age of $\geq 50$ years. These patients presented with SCC of the supraglottic larynx and hard palate and postoperative cancer of the tongue. All patients received radiotherapy between 2014 and 2019 as part of their standard care at our institution using the simultaneous integrated boost (SIB) technique. Computed tomography (CT) scans with 3-mm slice thickness for each patient were performed in head-first-supine position along with a standard HN thermoplast orfit mask on Somaton Sensation CT scanner (Siemens medical system, Malvern, PA, USA). After that, CT image sets were exported to the Monaco (Elekta Medical system, Crawley, UK) treatment planning system (TPS) in the Digital Imaging and Communication in Medi- 
cine format.

CT images were registered and fused with positron emission tomography and magnetic resonance imaging images to contour gross target volume. The clinical target volume and PTV were created using a margin of $12 \mathrm{~mm}$ and $5 \mathrm{~mm}$, respectively, according to the Radiation Therapy Oncology Group recommendations. Moreover, critical OARs were delineated on the CT images, namely, parotid glands, spinal cord, brainstem, larynx, mandible, and optic apparatus.

The dose prescriptions were 70 Gy (high-risk PTV), 63 Gy (intermediate-risk PTV), and 56 Gy (low-risk PTV) in 35 fractions for the supraglottic larynx; 70 Gy (high-risk PTV) and 56 Gy (low-risk PTV) in 35 fractions for the hard palate; and $60 \mathrm{~Gy}$ (tumor bed) and $54 \mathrm{~Gy}$ (nodal volumes) in 30 fractions for tongue cancer. According to the International Commission on Radiation Units and Measurements 83 [18], treatment plans were normalized in such a way that at least $95 \%$ of the PTV should cover $100 \%$ of the prescription dose. The dose objective for OARs were followed as prescribed by the Quantitative Analyses of Normal Tissue Effects in the Clinic group. Table 1 summarizes the planning dose objectives for the PTV and OARs. Treatment plans were generated using a 6-megavolt (MV) photon beam energized using a Synergy (Elekta Medical System) linear accelerator (linac). The Synergy linac is equipped with 40 pairs of MLC leaves with a width of $1 \mathrm{~cm}$ at the isocenter, which could deliver a maximum dose rate of $600 \mathrm{cGy} / \mathrm{min}$ at the dose maximum $\left(D_{\max }\right)$.

\section{Intensity-modulated radiation therapy planning}

Clinically acceptable dynamic MLC plans were generated on the Monaco TPS (version 5.11.02; Elekta Medical System). The Monaco TPS uses the Monte Carlo algorithm for optimization and final dose calculation, which is the gold standard presently. In Monaco, IMRT incorporates cost function-based segment shape optimization. Seven coplanar fields of 6-MV photon beam with couch and collimator angles at $0^{\circ}$ were used to design the respective plans. All gantry angles were equidistant from each other, that is, $0^{\circ}, 51^{\circ}, 102^{\circ}, 153^{\circ}, 204^{\circ}, 255^{\circ}$, and $306^{\circ}$, respectively. Additionally, a spill structure with a margin of $5 \mathrm{~mm}$ from the target was drawn to control low-dose spillage outside PTVs.
The recommended grid spacing of $3 \mathrm{~mm}$ and statistical uncertainty of $2 \%$ were employed for all treatment plans. The minimum segment size of $5 \mathrm{~mm}$ and maximum number of control point per field of 30 were chosen for optimization and dose calculation [19].

\section{Volumetric modulated arc therapy planning}

VMAT plans were created in the similar fashion as IMRT plans. All cost functions and dose objectives (target and OARs) were the same as those used in IMRT optimization. In VMAT planning, we used dual-arc ranges from $181^{\circ}$ to $179.9^{\circ}$ in clockwise and counterclockwise directions with an arc increment angle of $20^{\circ}$. The maximum number of control points per arc of 100 was chosen.

\section{Dosimetric evaluation}

Intercomparison of both the IMRT and VMAT plans was performed using analysis of dose-volume histogram (DVH) curves. Various dosimetric parameters were evaluated for the PTV and critical OARs using DVH data. The following dose indices were evaluated:

PTV: $\mathrm{D}_{95 \%}$ (dose to the $95 \%$ volume of the PTV), $\mathrm{D}_{98 \%}, \mathrm{D}_{50 \%}$, $\mathrm{D}_{2 \%}, \mathrm{D}_{\text {mean }}, \mathrm{D}_{\text {min }}$, and $\mathrm{D}_{\text {max }}$.

OARs: $\mathrm{D}_{\max }$ for serial organs, i.e., spinal cord, brainstem.

Mean dose for parallel organs, that is, parotid glands, lips, and larynx.

Low-dose volume analysis: $\mathrm{V}_{1 \mathrm{~Gy}}$ (volume of $1 \mathrm{~Gy}$ outside the target), $\mathrm{V}_{2 \mathrm{~Gy}}, \mathrm{~V}_{3 \mathrm{G} y}, \mathrm{~V}_{4 \mathrm{G} y}, \mathrm{~V}_{5 \mathrm{G} y}, \mathrm{~V}_{10 \mathrm{G} y}, \mathrm{~V}_{15 \mathrm{G} y}, \mathrm{~V}_{20 \mathrm{G} y}, \mathrm{~V}_{25 \mathrm{G} y}, \mathrm{~V}_{30 \mathrm{G} y}$, $D_{1 \%}$ (dose to the $1 \%$ volume outside the target), $D_{2 \%}, D_{5 \%}$.

Table 1. Planning dose objectives for the planning target volume (PTV) and organs-at-risk

\begin{tabular}{lcl}
\hline \multicolumn{1}{c}{ Structure } & Parameter & \multicolumn{1}{c}{ Plan objective } \\
\hline PTV & $\mathrm{D}_{95 \%}$ & $=100 \%$ of prescribed dose \\
& $\mathrm{D}_{110 \%}$ & $<10 \%$ of PTV \\
& $\mathrm{D}_{93 \%}$ & $<1 \%$ of PTV \\
Spinal cord & $\mathrm{D}_{\max }$ & $<45 \mathrm{~Gy}$ \\
Brainstem & $\mathrm{D}_{\max }$ & $<54 \mathrm{~Gy}$ \\
Lips & $\mathrm{D}_{\text {mean }}$ & $<35 \mathrm{~Gy}$ \\
Contralateral parotid & $\mathrm{D}_{\text {mean }}$ & $<26 \mathrm{~Gy}$ \\
Larynx & $\mathrm{D}_{\text {mean }}$ & $<50 \mathrm{~Gy}$ \\
Mandible & $\mathrm{D}_{\text {max }}$ & $<75 \mathrm{~Gy}$ \\
\hline
\end{tabular}


Additionally, a ring structure was delineated to be $5 \mathrm{~cm}$ beyond the PTV to estimate the mean dose to the normal tissues lying in that region.

Moreover, the UI, CI, HI, conformity number $(\mathrm{CN})$, and normal tissue integral dose (NTID) were computed using the following formulas:

$$
\begin{gathered}
\mathrm{UI}=\frac{\mathrm{D}_{5 \%}(\mathrm{~Gy})}{\mathrm{D}_{95 \%}(\mathrm{~Gy})} \\
\mathrm{CI}=\frac{\mathrm{TV}_{95 \%}(\mathrm{cc})}{\mathrm{TV}} \\
\mathrm{HI}=\frac{\mathrm{D}_{2 \%}(\mathrm{~Gy})-\mathrm{D}_{98 \%}(\mathrm{~Gy})}{\mathrm{D}_{50 \%}(\mathrm{~Gy})} \\
\mathrm{CN}=\left(\frac{\mathrm{TV}_{\mathrm{RI}}}{\mathrm{TV}}\right) \times\left(\frac{\mathrm{TV}}{\mathrm{V}_{\mathrm{RI}}}\right)
\end{gathered}
$$

NTID=mean dose $x$

volume of normal tissues outside the PTV

where TV stands for the target volume in $\mathrm{cm}^{3}, \mathrm{TV}_{\mathrm{RI}}$ stands for the volume covered by the reference isodose, and $\mathrm{V}_{\mathrm{RI}}$ stands for the volume of the prescription isodose outside the target volume (inside body).

\section{Statistical analysis}

The dosimetric difference between IMRT and VMAT was analyzed using Statistical Package for the Social Sciences (version 20; IBM Corp., Armonk, NY, USA) in terms of the mean, standard deviation, and $P$-values. The independent paired t-test with a confidence interval limit of $95 \%$ was performed to assess the dosimetric endpoints for the target and OARs. $P$-values of less than 0.05 were used to denote statistical significance.

\section{Quality assurance}

Moreover, patient-specific quality assurance (PSQA) was executed using ArcCHECK (Sun Nuclear, Melbourne, FL, USA), which is a $3 \mathrm{D}$ cylindrical array of 1,386 equally spaced (10 $\mathrm{mm}$ apart) diode detectors. All treatment plans were calculated, and the same plans were delivered on ArcCHECK. SNC (version 6.2; Sun Nuclear) was used for gamma analysis. A criterion of 3-mm distance to agreement
(DTA) and 3\% dose difference (DD) with a threshold value of $10 \%$ was used for PSQA analysis. Additionally, 2-mm DTA and $2 \%$ DD criteria with a threshold value of $10 \%$ was examined for gamma analysis. A tolerance of $\pm 5 \%$ was considered acceptable between the ion-chamber-measured and TPS-calculated doses [20].

\section{Results}

Clinically acceptable treatment plans were created using the IMRT and VMAT techniques for all patients. Qualitative and quantitative analyses were performed on dose distribution created for IMRT and VMAT plans. The data were derived from cumulative DVH data for each treatment plan. Table 2 and 3 illustrate the dosimetric data on the PTV and OARs for cancers of the supraglottic larynx, hard palate, and tongue using the IMRT and VMAT techniques. Fig. 1 shows the dose distribution in axial and coronal planes using IMRT and VMAT techniques along their DVH.

No significant $(P>0.05)$ difference in PTV coverage $\left(D_{95 \%}\right)$ was observed between IMRT and VMAT plans for cancers of the supraglottic larynx, hard palate, and tongue. A noticeable increase $(P<0.05)$ in $\mathrm{D}_{50 \%}$ was observed for VMAT plans compared with that for IMRT plans for cancers of the supraglottic larynx, hard palate, and tongue. Moreover, a slight increase in the target mean dose and $\mathrm{D}_{\max }$ was observed for VMAT compared with those for IMRT for cancers of the supraglottic larynx, hard palate, and tongue, except for VMAT $\mathrm{PTV}_{56}$ for supraglottic larynx cancer. A significant $(P<0.05)$ increase in UI was observed for VMAT plans compared with that for IMRT plans, except for PTV $\mathrm{PT}_{63}$ and $\mathrm{PTV}_{56}$ for supraglottic larynx cancer. Additionally, significant $(P<0.05)$ reductions in $\mathrm{HI}$ were observed for VMAT plans compared with that for IMRT plans, except for $\operatorname{PTV}_{56}(P>0.05)$ for supraglottic larynx cancer. However, no significant $(P>0.05)$ difference in CI was found between VMAT and IMRT plans. Additionally, a significant $(P>0.05)$ increase in the $\mathrm{CN}$ number was noticed for VMAT plans compared with that for IMRT for all cases.

A slight increase in OAR dose was noted for VMAT plans compared with that for IMRT plans for all cases, except for the brainstem in tongue cancer (Table 3 ). Table 4 shows the increase in monitor units (MUs) for VMAT plans compared 
Table 2. The dosimetric parameters for PTV in supraglottic larynx, hard palate, and tongue cancers using the IMRT and VMAT techniques

\begin{tabular}{|c|c|c|c|c|c|c|c|c|c|c|}
\hline \multirow{2}{*}{ Target } & \multirow{2}{*}{ Parameter } & \multicolumn{2}{|c|}{ Supraglottic larynx } & \multicolumn{2}{|c|}{ Hard palate } & \multicolumn{2}{|c|}{ Tongue } & \multicolumn{3}{|c|}{$P$-value } \\
\hline & & IMRT & VMAT & IMRT & VMAT & IMRT & VMAT & Supra & HP & Tongue \\
\hline \multirow[t]{11}{*}{$\mathrm{PTV}_{70}$} & $\mathrm{D}_{95 \%}(\mathrm{~Gy})$ & 70.00 & 70.00 & 70.00 & 70.00 & & & & & \\
\hline & $\mathrm{D}_{98 \%}(\mathrm{~Gy})$ & $69.20 \pm 0.16$ & $69.07 \pm 0.15$ & $69.17 \pm 0.08$ & $68.98 \pm 0.16$ & & & 0.011 & 0.006 & \\
\hline & $\mathrm{D}_{50 \%}(\mathrm{~Gy})$ & $72.66 \pm 0.34$ & $73.02 \pm 0.42$ & $72.48 \pm 0.28$ & $73.10 \pm 0.36$ & & & 0.009 & 0.001 & \\
\hline & $\mathrm{D}_{2 \%}(\mathrm{~Gy})$ & $75.38 \pm 0.52$ & $76.13 \pm 0.61$ & $75.14 \pm 0.51$ & $76.31 \pm 0.75$ & & & 0.019 & 0.001 & \\
\hline & Mean (Gy) & $72.04 \pm 1.07$ & $72.29 \pm 1.23$ & $71.99 \pm 0.34$ & $73.01 \pm 0.34$ & & & 0.548 & 0.045 & \\
\hline & $\mathrm{D}_{\max }(\mathrm{Gy})$ & $78.54 \pm 1.24$ & $79.73 \pm 0.96$ & $78.60 \pm 1.17$ & $80.77 \pm 0.93$ & & & 0.007 & 0.001 & \\
\hline & $\mathrm{D}_{\min }(\mathrm{Gy})$ & $63.31 \pm 2.19$ & $62.11 \pm 2.25$ & $62.77 \pm 1.74$ & $61.57 \pm 2.15$ & & & 0.131 & 0.081 & \\
\hline & UI & $1.07 \pm 0.01$ & $1.08 \pm 0.01$ & $1.06 \pm 0.01$ & $1.08 \pm 0.01$ & & & $<0.001$ & $<0.001$ & \\
\hline & $\mathrm{HI}$ & $0.072 \pm 0.01$ & $0.071 \pm 0.01$ & $0.081 \pm 0.01$ & $0.078 \pm 0.01$ & & & $<0.001$ & $<0.001$ & \\
\hline & $\mathrm{CI}$ & $0.99 \pm 0.03$ & $0.99 \pm 0.03$ & $0.99 \pm 0.01$ & $1.00 \pm 0.01$ & & & 0.791 & 0.093 & \\
\hline & $\mathrm{CN}$ & $0.88 \pm 0.03$ & $0.90 \pm 0.03$ & $0.92 \pm 0.03$ & $0.93 \pm 0.03$ & & & 0.972 & 0.667 & \\
\hline \multirow[t]{11}{*}{$\mathrm{PTV}_{63}$} & $\mathrm{D}_{95 \%}(\mathrm{~Gy})$ & $64.01 \pm 0.91$ & $63.91 \pm 1.18$ & & & & & 0.511 & & \\
\hline & $\mathrm{D}_{98 \%}(\mathrm{~Gy})$ & $62.95 \pm 0.98$ & $62.67 \pm 1.45$ & & & & & 0.197 & & \\
\hline & $\mathrm{D}_{50 \%}(\mathrm{~Gy})$ & $68.65 \pm 1.52$ & $69.24 \pm 1.46$ & & & & & 0.001 & & \\
\hline & $\mathrm{D}_{2 \%}(\mathrm{~Gy})$ & $74.69 \pm 0.79$ & $75.26 \pm 0.79$ & & & & & 0.006 & & \\
\hline & Mean (Gy) & $66.06 \pm 1.06$ & $66.19 \pm 1.62$ & & & & & 0.767 & & \\
\hline & $\mathrm{D}_{\max }(\mathrm{Gy})$ & $74.65 \pm 1.28$ & $75.29 \pm 0.96$ & & & & & 0.192 & & \\
\hline & $\mathrm{D}_{\min }(\mathrm{Gy})$ & $51.02 \pm 3.53$ & $51.35 \pm 4.05$ & & & & & 0.717 & & \\
\hline & UI & $1.15 \pm 0.01$ & $1.16 \pm 0.02$ & & & & & 0.102 & & \\
\hline & $\mathrm{HI}$ & $0.171 \pm 0.01$ & $0.168 \pm 0.02$ & & & & & 0.032 & & \\
\hline & $\mathrm{CI}$ & $0.99 \pm 0.03$ & $0.98 \pm 0.03$ & & & & & 0.341 & & \\
\hline & $\mathrm{CN}$ & $0.86 \pm 0.05$ & $0.88 \pm 0.06$ & & & & & 0.142 & & \\
\hline \multirow[t]{11}{*}{$\mathrm{PTV}_{56}$} & $\mathrm{D}_{95 \%}(\mathrm{~Gy})$ & $57.78 \pm 0.92$ & $57.82 \pm 1.04$ & $58.83 \pm 0.83$ & $59.70 \pm 1.11$ & & & 0.771 & 0.055 & \\
\hline & $\mathrm{D}_{98 \%}(\mathrm{~Gy})$ & $56.94 \pm 0.82$ & $56.79 \pm 1.03$ & $57.80 \pm 0.72$ & $58.25 \pm 0.94$ & & & 0.423 & 0.066 & \\
\hline & $\mathrm{D}_{50 \%}(\mathrm{~Gy})$ & $63.75 \pm 3.64$ & $64.28 \pm 3.72$ & $67.40 \pm 3.33$ & $68.29 \pm 2.72$ & & & 0.003 & 0.011 & \\
\hline & $\mathrm{D}_{2 \%}(\mathrm{~Gy})$ & $70.63 \pm 5.71$ & $71.34 \pm 5.62$ & $74.12 \pm 1.53$ & $75.13 \pm 1.77$ & & & 0.002 & 0.002 & \\
\hline & Mean (Gy) & $58.93 \pm 1.17$ & $59.04 \pm 1.47$ & $62.51 \pm 0.91$ & $64.11 \pm 1.09$ & & & 0.769 & 0.002 & \\
\hline & $\mathrm{D}_{\max }(\mathrm{Gy})$ & $71.35 \pm 2.50$ & $70.92 \pm 2.73$ & $75.61 \pm 1.14$ & $76.55 \pm 0.99$ & & & 0.407 & 0.008 & \\
\hline & $\mathrm{D}_{\min }(\mathrm{Gy})$ & $46.54 \pm 3.60$ & $45.82 \pm 2.89$ & $45.65 \pm 3.28$ & $46.42 \pm 1.79$ & & & 0.378 & 0.518 & \\
\hline & UI & $1.21 \pm 0.09$ & $1.11 \pm 0.37$ & $1.24 \pm 0.03$ & $1.24 \pm 0.04$ & & & 0.391 & 0.576 & \\
\hline & $\mathrm{HI}$ & $0.218 \pm 0.08$ & $0.214 \pm 0.07$ & $0.232 \pm 0.02$ & $0.229 \pm 0.03$ & & & 0.051 & 0.302 & \\
\hline & $\mathrm{CI}$ & $1.00 \pm 0.01$ & $1.00 \pm 0.01$ & $1.00 \pm 0.01$ & $0.99 \pm 0.01$ & & & 0.892 & 0.271 & \\
\hline & $\mathrm{CN}$ & $0.72 \pm 0.21$ & $0.75 \pm 0.21$ & $0.90 \pm 0.11$ & $0.92 \pm 0.12$ & & & $<0.001$ & 0.113 & \\
\hline \multirow[t]{11}{*}{$\mathrm{PTV}_{60}$} & $\mathrm{D}_{95 \%}(\mathrm{~Gy})$ & & & & & 60.00 & 60.00 & & & \\
\hline & $\mathrm{D}_{98 \%}(\mathrm{~Gy})$ & & & & & $58.93 \pm 0.18$ & $58.76 \pm 0.25$ & & & $<0.001$ \\
\hline & $\mathrm{D}_{50 \%}(\mathrm{~Gy})$ & & & & & $62.84 \pm 0.34$ & $63.51 \pm 0.45$ & & & $<0.001$ \\
\hline & $\mathrm{D}_{2 \%}(\mathrm{~Gy})$ & & & & & $65.44 \pm 0.53$ & $66.81 \pm 0.74$ & & & $<0.001$ \\
\hline & Mean (Gy) & & & & & $62.69 \pm 0.31$ & $63.35 \pm 0.41$ & & & $<0.001$ \\
\hline & $\mathrm{D}_{\max }(\mathrm{Gy})$ & & & & & $69.43 \pm 1.13$ & $71.67 \pm 1.19$ & & & $<0.001$ \\
\hline & $\mathrm{D}_{\min }(\mathrm{Gy})$ & & & & & $50.49 \pm 2.27$ & $49.72 \pm 3.02$ & & & 0.871 \\
\hline & UI & & & & & $1.08 \pm 0.01$ & $1.1 \pm 0.01$ & & & $<0.001$ \\
\hline & $\mathrm{HI}$ & & & & & $0.126 \pm 0.01$ & $0.123 \pm 0.01$ & & & $<0.001$ \\
\hline & $\mathrm{CI}$ & & & & & $0.99 \pm 0.001$ & $0.99 \pm 0.01$ & & & 0.244 \\
\hline & $\mathrm{CN}$ & & & & & $0.88 \pm 0.04$ & $0.89 \pm 0.04$ & & & 0.478 \\
\hline
\end{tabular}


Table 2. Continued

\begin{tabular}{|c|c|c|c|c|c|c|c|c|c|c|}
\hline \multirow{2}{*}{ Target } & \multirow{2}{*}{ Parameter } & \multicolumn{2}{|c|}{ Supraglottic larynx } & \multicolumn{2}{|c|}{ Hard palate } & \multicolumn{2}{|c|}{ Tongue } & \multicolumn{3}{|c|}{$P$-value } \\
\hline & & IMRT & VMAT & IMRT & VMAT & IMRT & VMAT & Supra & HP & Tongue \\
\hline \multirow[t]{11}{*}{$\mathrm{PTV}_{54}$} & $\mathrm{D}_{95 \%}(\mathrm{~Gy})$ & & & & & $54.69 \pm 0.43$ & $54.48 \pm 0.53$ & & & 0.072 \\
\hline & $\mathrm{D}_{98 \%}(\mathrm{~Gy})$ & & & & & $53.89 \pm 0.54$ & $53.38 \pm 0.58$ & & & 0.001 \\
\hline & $\mathrm{D}_{50 \%}(\mathrm{~Gy})$ & & & & & $57.23 \pm 1.31$ & $57.91 \pm 1.38$ & & & 0.001 \\
\hline & $\mathrm{D}_{2 \%}(\mathrm{~Gy})$ & & & & & $61.50 \pm 2.78$ & $62.69 \pm 2.90$ & & & 0.001 \\
\hline & Mean (Gy) & & & & & $56.81 \pm 0.90$ & $57.08 \pm 0.59$ & & & 0.228 \\
\hline & $\mathrm{D}_{\max }(\mathrm{Gy})$ & & & & & $64.16 \pm 1.42$ & $64.76 \pm 0.99$ & & & 0.046 \\
\hline & $\mathrm{D}_{\min }(\mathrm{Gy})$ & & & & & $43.06 \pm 4.12$ & $43.93 \pm 2.89$ & & & 0.115 \\
\hline & UI & & & & & $1.10 \pm 0.05$ & $1.13 \pm 0.05$ & & & $<0.001$ \\
\hline & HI & & & & & $0.161 \pm 0.05$ & $0.159 \pm 0.05$ & & & $<0.001$ \\
\hline & CI & & & & & $1.00 \pm 0.001$ & $0.99 \pm 0.01$ & & & 0.078 \\
\hline & $\mathrm{CN}$ & & & & & $0.69 \pm 0.18$ & $0.72 \pm 0.18$ & & & 0.101 \\
\hline
\end{tabular}

Values are presented in mean \pm standard deviation.

IMRT, intensity-modulated radiation therapy; VMAT, volumetric modulated arc therapy; PTV, planning target volume; UI, uniformity index; HI, homogeneity index; $\mathrm{CI}$, conformity index; $\mathrm{CN}$, conformation number.

Table 3. The dosimetric parameters for OARs in supraglottic larynx, hard palate, and tongue cancers using the IMRT and VMAT techniques

\begin{tabular}{|c|c|c|c|c|c|}
\hline Site & OARs & Parameter (Gy) & IMRT & VMAT & $P$-value \\
\hline \multirow[t]{8}{*}{ Supraglottic larynx } & Contra lateral parotid & $\mathrm{D}_{\text {mean }}$ & $22.76 \pm 1.24$ & $23.41 \pm 1.21$ & $<0.001$ \\
\hline & & $\mathrm{D}_{50 \%}$ & $18.22 \pm 2.28$ & $18.31 \pm 0.81$ & 0.853 \\
\hline & Brainstem & $\mathrm{D}_{\max }$ & $33.88 \pm 2.57$ & $36.21 \pm 2.53$ & $<0.001$ \\
\hline & Spinal cord & $\mathrm{D}_{\max }$ & $32.77 \pm 1.63$ & $33.92 \pm 1.58$ & 0.009 \\
\hline & Lips & $\mathrm{D}_{\text {mean }}$ & $32.71 \pm 3.74$ & $34.65 \pm 2.96$ & 0.016 \\
\hline & Larynx & $\mathrm{D}_{\text {mean }}$ & $49.68 \pm 1.91$ & $50.47 \pm 1.52$ & 0.004 \\
\hline & Mandible & $\mathrm{D}_{\max }$ & $75.83 \pm 2.61$ & $76.46 \pm 2.19$ & 0.102 \\
\hline & Ring ( $5 \mathrm{~cm}$ beyond PTV) & $\mathrm{D}_{\text {mean }}$ & $35.89 \pm 3.32$ & $35.48 \pm 3.04$ & 0.301 \\
\hline \multirow[t]{8}{*}{ Hard palate } & Contra lateral parotid & $\mathrm{D}_{\text {mean }}$ & $24.64 \pm 2.37$ & $25.43 \pm 2.69$ & 0.059 \\
\hline & & $\mathrm{D}_{50 \%}$ & $20.24 \pm 0.96$ & $21.75 \pm 0.75$ & 0.001 \\
\hline & Brainstem & $\mathrm{D}_{\max }$ & $34.69 \pm 3.65$ & $35.68 \pm 3.05$ & 0.536 \\
\hline & Spinal cord & $\mathrm{D}_{\max }$ & $34.54 \pm 2.35$ & $36.38 \pm 2.36$ & 0.029 \\
\hline & Lips & $\mathrm{D}_{\text {mean }}$ & $34.72 \pm 1.91$ & $35.86 \pm 1.57$ & 0.093 \\
\hline & Larynx & $\mathrm{D}_{\text {mean }}$ & $47.84 \pm 1.04$ & $49.13 \pm 0.82$ & 0.011 \\
\hline & Mandible & $\mathrm{D}_{\max }$ & $77.02 \pm 1.84$ & $77.32 \pm 1.99$ & 0.394 \\
\hline & Ring ( $5 \mathrm{~cm}$ beyond PTV) & $\mathrm{D}_{\text {mean }}$ & $36.44 \pm 3.79$ & $35.72 \pm 4.52$ & 0.062 \\
\hline \multirow[t]{8}{*}{ Tongue } & Contra lateral parotid & $\mathrm{D}_{\text {mean }}$ & $23.61 \pm 1.71$ & $24.76 \pm 1.76$ & $<0.001$ \\
\hline & & $\mathrm{D}_{50 \%}$ & $19.09 \pm 1.76$ & $20.77 \pm 1.65$ & $<0.001$ \\
\hline & Brainstem & $\mathrm{D}_{\max }$ & $30.64 \pm 3.29$ & $30.23 \pm 3.87$ & 0.671 \\
\hline & Spinal cord & $\mathrm{D}_{\max }$ & $35.44 \pm 1.76$ & $35.65 \pm 2.25$ & 0.656 \\
\hline & Lips & $\mathrm{D}_{\text {mean }}$ & $34.59 \pm 2.41$ & $35.33 \pm 1.96$ & 0.206 \\
\hline & Larynx & $\mathrm{D}_{\text {mean }}$ & $48.46 \pm 1.66$ & $48.71 \pm 2.55$ & 0.527 \\
\hline & Mandible & $\mathrm{D}_{\max }$ & $67.41 \pm 0.94$ & $68.78 \pm 1.28$ & $<0.001$ \\
\hline & Ring ( $5 \mathrm{~cm}$ beyond PTV) & $\mathrm{D}_{\text {mean }}$ & $32.10 \pm 1.58$ & $31.38 \pm 1.71$ & 0.001 \\
\hline
\end{tabular}

Values are presented as mean \pm standard deviation.

OARs, organs-at-risk; IMRT, intensity-modulated radiation therapy; VMAT, volumetric modulated arc therapy; PTV, planning target volume. 

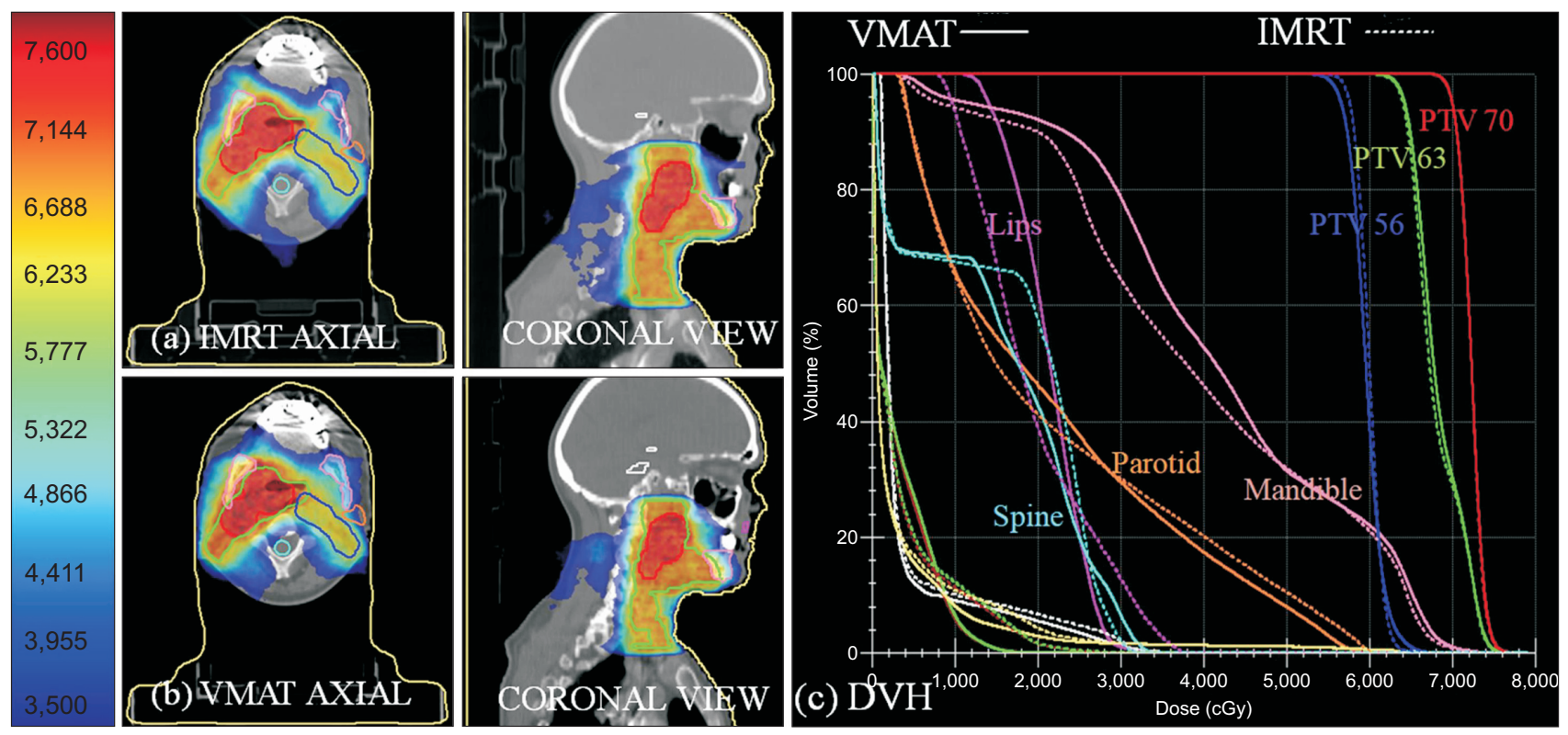

Fig. 1. Comparison of dose distribution in axial and coronal planes using (a) the IMRT and (b) VMAT techniques along with their (c) DVH. IMRT, intensity-modulated radiation therapy; VMAT, volumetric modulated arc therapy; DVH, dose-volume histograms; PTV, planning target volume.

Table 4. The MUs and NTID in supraglottic larynx, hard palate, and tongue cancers using the IMRT and VMAT techniques

\begin{tabular}{|c|c|c|c|c|c|c|}
\hline \multirow{2}{*}{ Site } & \multicolumn{3}{|c|}{ MUs } & \multicolumn{3}{|c|}{ NTID } \\
\hline & IMRT & VMAT & $P$-value & IMRT & VMAT & $P$-value \\
\hline Supraglottic larynx & $871.59 \pm 66.70$ & $875.64 \pm 84.56$ & 0.971 & $3.77 \pm 1.08$ & $3.60 \pm 1.08$ & 0.003 \\
\hline Hard palate & $854.57 \pm 80.32$ & $876.35 \pm 88.65$ & 0.307 & $4.53 \pm 1.83$ & $3.95 \pm 1.35$ & 0.025 \\
\hline Tongue & $915.35 \pm 79.48$ & $984.64 \pm 123.01$ & $<0.001$ & $3.35 \pm 1.09$ & $3.09 \pm 0.91$ & $<0.001$ \\
\hline
\end{tabular}

Values are presented as mean \pm standard deviation.

MU, monitor unit; NTID, integral dose to normal tissue; IMRT, intensity-modulated radiation therapy; VMAT, volumetric modulated arc therapy.

with those for IMRT plans in cancers of the supraglottic larynx $(P>0.05,0.46 \%)$, hard palate $(P>0.05,2.54 \%)$, and tongue $(P<0.05,7.56 \%)$. Additionally, significant $(P<0.05)$ reductions in the NTID were noted for VMAT plans compared with that for IMRT plans in cancers of the supraglottic larynx (4.50\%), hard palate (12.80\%), and tongue (7.76\%). Furthermore, a reduction in the mean dose to the ring structure (i.e., $5 \mathrm{~cm}$ beyond the PTV) was observed using the VMAT technique compared with that using the IMRT technique for cancers of the supraglottic larynx (1.14\%, $P>0.05)$, hard palate $(1.97 \%, P>0.05)$, and tongue $(2.24 \%$, $P<0.05)$.

In low-dose volume evaluation, the doses to the $1 \%, 2 \%$, and $5 \%$ volumes of normal tissue were evaluated and recorded in Table 5. A slight reduction $(P>0.05)$ in $\mathrm{D}_{5 \%}$ was ob- served for VMAT plans compared with that for IMRT plans for all HNC cases. Additionally, a reduction in dose volumes ranging from 1 Gy to 30 Gy was observed for VMAT plans compared with IMRT plans, except for $\mathrm{V}_{1 \mathrm{~Gy}}, \mathrm{~V}_{2 \mathrm{~Gy}}$, and $\mathrm{V}_{30 \mathrm{~Gy}}$ for supraglottic larynx cancer and $V_{1 G y}$ for tongue cancer.

The PSQA analysis showed comparable gamma passing rates for both techniques. Point dose analysis results were within $\pm 5 \%$ for all cases using the IMRT and VMAT techniques. The gamma passing rate (with $3 \mathrm{~mm} / 3 \%$ ) in the relative dose mode was $97.11 \% \pm 1.69 \%$ and $97.44 \% \pm 1.80 \%$, whereas, in the absolute dose mode, the gamma passing rate was $96.45 \% \pm 0.84 \%$ and $96.59 \% \pm 2.13 \%$ for the IMRT and VMAT techniques, respectively. The gamma passing rate (with $2 \mathrm{~mm} / 2 \%$ ) rate in the relative dose mode was $88.46 \% \pm 1.24 \%$ and $90.29 \% \pm 2.12 \%$, whereas, in the absolute 


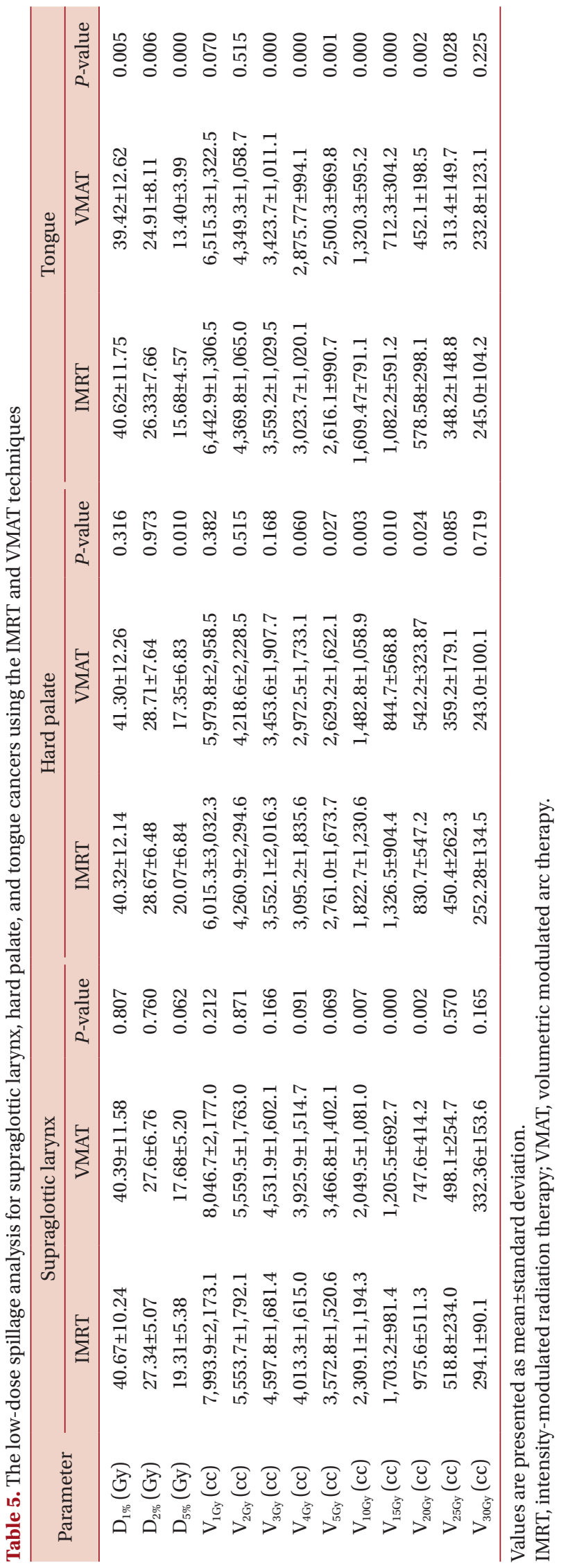

dose mode, the gamma passing rate was $85.57 \% \pm 1.91 \%$ and $87.71 \% \pm 2.79 \%$ for the IMRT and VMAT techniques, respectively. Fig. 2 shows the gamma analysis of a representative patient: ArcCHECK-measured plan, TPS-calculated plan, difference between ArcCHECK-measured and TPScalculated plans, and measured points that matched the acceptance criteria.

\section{Discussion}

This study analyzed the dosimetric difference between seven-field IMRT and double-arc VMAT treatment plans in supraglottic larynx, hard palate, and tongue cancers. The results revealed no significant difference in target coverage between IMRT and VMAT plans. Additionally, IMRT offers a slightly better OAR-sparing ability than VMAT; however, IMRT had CI, UI, and CN comparable to those of VMAT, but its HI was inferior to that of VMAT. However, VMAT offers less NTID, mean dose to ring structure (i.e., $5 \mathrm{~cm}$ beyond the PTV), and dose volumes ranging from 1 Gy to $30 \mathrm{~Gy}$ to normal tissues than IMRT.

Radhakrishana et al. [21] have demonstrated the comparable target coverage for the IMRT and VMAT techniques using the Monaco TPS for nasopharyngeal carcinoma using the SIB technique. In their study, VMAT has shown a more conformal dose distribution with a better or comparable OAR-sparing ability.

This study revealed that VMAT required more MUs than IMRT in HNC. Moreover, Nithya et al. [22] have reported similar findings in a comparative study between VMAT and IMRT for tongue cancer using the Monaco TPS. Additionally, Nithya et al. [22] have highlighted a dip in the literature about comparative studies for HNC using the Monaco TPS compared with Eclipse (Varian Medical System, Palo Alto, CA, USA) and other TPS. Several studies have reported that VMAT requires lesser MUs than IMRT for HNC [21,22]. HNC planning requires a high degree of modulation for a clinically acceptable treatment plan. Additionally, HNC presents a unique challenge of concave dose distribution along with close proximity of OARs to the target volume. The number of MUs depends on the size of the target and the degree of modulation employed in the optimization process for OAR sparing [23]. 
a $\mathrm{ArCCHECK}$ (measured)

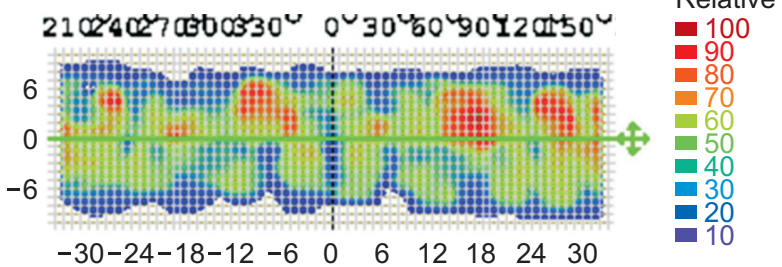

b TPS plan (calculated)

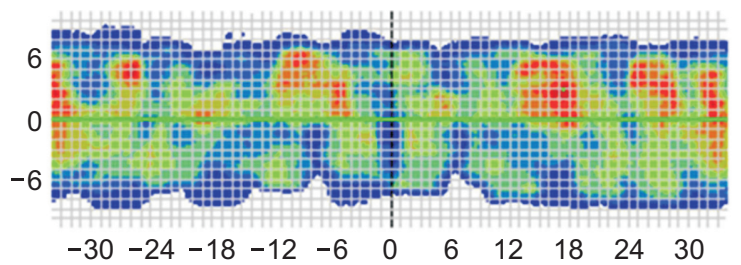

Relative

- 100

- 90

- 80

- 70

- 60

50
-40

40
-30

- 20

C ArcCHECK-TPS plan

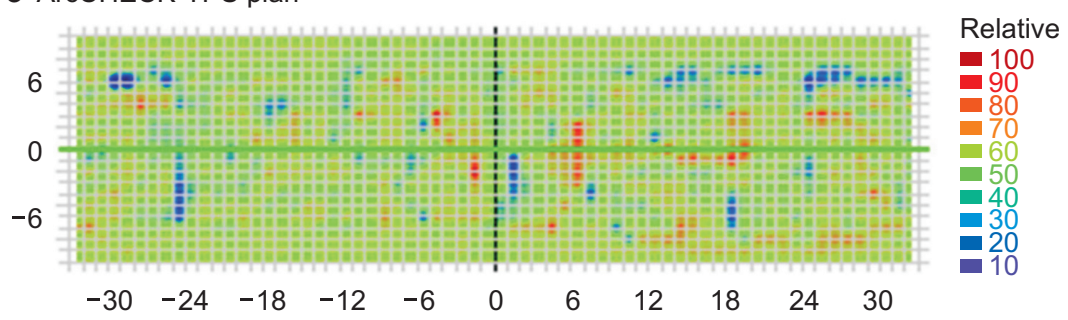

d

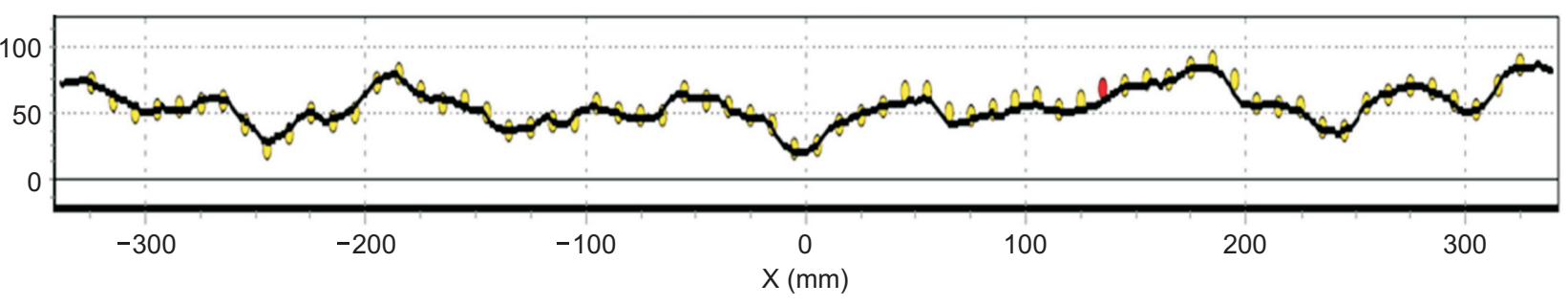

Fig. 2. Gamma analysis of a representative patient. (a) ArcCHECK-measured plan, (b) TPS-calculated plan, (c) difference between the ArcCHECK-measured and TPS-calculated plans, and (d) measured points that matched the acceptance criteria were shown in yellow color and points that don't match acceptance criteria were shown in red color. TPS, treatment planning system.

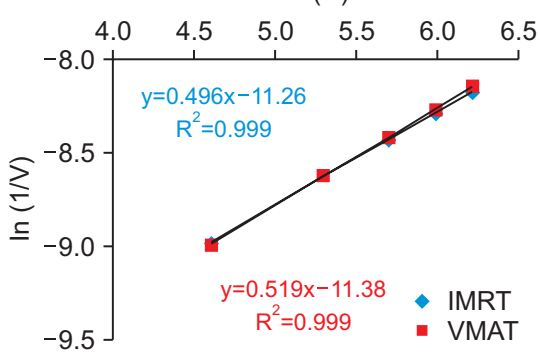

b

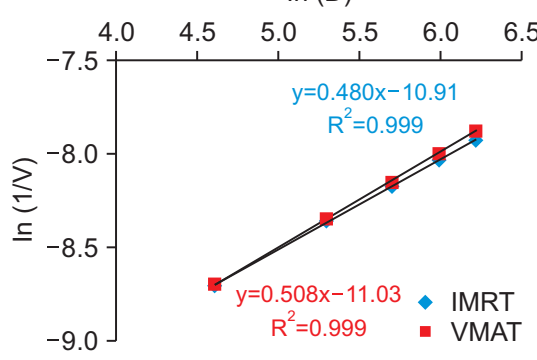

C $\quad \ln (\mathrm{D})$

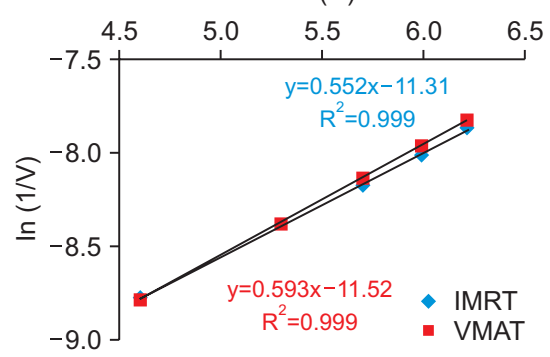

Fig. 3. The rate of dose falloff beyond the target by plotting $\ln (\mathrm{D})$ versus $\ln (1 / \mathrm{V})$ for (a) supraglottic larynx, (b) hard palate, and (c) tongue cancers. IMRT, intensity-modulated radiation therapy; VMAT, volumetric modulated arc therapy.

This study revealed that VMAT offers lesser NTID, mean dose to ring structure (i.e., $5 \mathrm{~cm}$ beyond the PTV), and dose volumes (ranging from $1 \mathrm{~Gy}$ to $30 \mathrm{~Gy}$ ) to normal tissues than IMRT. The dose outside the treatment field depends on the target size and increases with an increase in the target volume because an increase in the target volume leads to more irradiation of the tissues along the target length [24]. The scatter contribution from the collimator and patient body along with softer energy spectrum outside the treatment field (compared with within the treatment field) plays a vital role in the irradiation dose to normal tissues outside the target volume [25,26]. Moreover, Fig. 3 shows a gradual decrease in the mean volume (V) encompassed by a certain dose (D) on a logarithmic scale for an increase in the dose and reveals that VMAT plans have a higher dose falloff than IMRT plans for supraglottic larynx, hard palate, and tongue 
cancers. This can be because VMAT offers more freedom in the optimization process restricting radiation doses to nearby OARs and normal tissues outside the target.

Hall [27] and Hall and Wuu [28] advocated that a lowdose volume may not cause acute or subacute clinical morbidity but could be carcinogenic and reported that IMRT probably has a $1 \%$ to $1.75 \%$ higher risk of secondary malignancies than conventional radiotherapy in patients surviving for 10 years. Followill et al. [29] have reported an increased risk of secondary malignancies by $0.4 \%$ to $1.0 \%$ with IMRT compared with conventional RT techniques. Hauri and Schneider [30] have evaluated the impact of treatment techniques on out-of-field low-dose volumes and recommended VMAT over IMRT in terms of radiological protection to patients with cancer. Because VMAT requires less beam-on time than IMRT leads, a reduction in the outof-field dose is expected.

Kry et al. [31] have reported that secondary malignancy is more likely to occur in areas surrounding the target receiving doses greater than 3 Gy. However, Diallo et al. [15] have reported that areas receiving doses smaller than 2.5 Gy are the prominent areas for the occurrence of secondary malignancies. In external beam radiotherapy, a larger area around the target gets exposed to radiation. Targets are exposed to a high dose of radiation to maintain the therapeutic level, and surrounding areas should be considered in plan evaluation to minimize exposure to radiation. Therefore, low-dose volumes should be carefully evaluated for the risk of secondary malignancies and for plan optimization for better consideration of nearby tissues in planning.

\section{Conclusions}

For advanced-stage HNCs, both IMRT and VMAT offer satisfactory clinical plans. For similar target coverage, VMAT offers a conformal and homogeneous dose distribution with a comparable OAR-sparing ability and a higher dose falloff outside the target volume compared with IMRT, which provides an edge over IMRT in reducing the risk of secondary malignancies.

\section{Acknowledgements}

The authors thank the management of Rajiv Gandhi Cancer Institute \& Research Centre in New Delhi, India, for their continued support and encouragement to complete this research work.

\section{Conflicts of Interest}

The authors have nothing to disclose.

\section{Availability of Data and Materials}

The data that support the findings of this study are available on request from the corresponding author.

\section{Author Contributions}

Conceptualization: Gourav Kumar and Lalit Kumar. Data curation: Gourav Kumar. Formal analysis: Manindra Bhushan. Funding acquisition: None. Investigation: Pawan Kumar and Soumitra Barik. Methodology: Sandeep Purohit. Project administration: Vimal Kishore. Resources: Soumitra Barik. Software: Sandeep Purohit and Pawan Kumar. Supervision: Manindra Bhushan and Vimal Kishore. Validation: Pawan Kumar and Kothanda Raman. Visualization: Manindra Bhushan. Writing-original draft: Gourav Kumar. Writing-review \& editing: Lalit Kumar.

\section{References}

1. Bhattacharjee A, Chakraborty A, Purkaystha P. Prevalence of head and neck cancers in the North East-an institutional study. Indian J Otolaryngol Head Neck Surg. 2006;58:15-19.

2. Sharma JD, Baishya N, Kataki AC, Kalita CR, Das AK, Rahman T. Head and neck squamous cell carcinoma in young adults: a hospital-based study. Indian J Med Paediatr Oncol. 2019;40(S1):S18-S22.

3. Grégoire V, Lefebvre JL, Licitra L, Felip E; EHNS-ESMOESTRO Guidelines Working Group. Squamous cell carcinoma of the head and neck: EHNS-ESMO-ESTRO Clinical Practice Guidelines for diagnosis, treatment and follow-up. Ann Oncol. 2010;21 Suppl 5:v184-v186. 
4. Vigneswaran N, Williams MD. Epidemiologic trends in head and neck cancer and aids in diagnosis. Oral Maxillofac Surg Clin North Am. 2014;26:123-141.

5. Bray F, Ferlay J, Soerjomataram I, Siegel RL, Torre LA, Jemal A. Global cancer statistics 2018: GLOBOCAN estimates of incidence and mortality worldwide for 36 cancers in 185 countries. CA Cancer J Clin. 2018;68:394-424.

6. Gupta T, Agarwal J, Jain S, Phurailatpam R, Kannan S, Ghosh-Laskar S, et al. Three-dimensional conformal radiotherapy (3D-CRT) versus intensity modulated radiation therapy (IMRT) in squamous cell carcinoma of the head and neck: a randomized controlled trial. Radiother Oncol. 2012;104:343-348.

7. Ge X, Liao Z, Yuan J, Mao D, Li Y, Yu E, et al. Radiotherapyrelated quality of life in patients with head and neck cancers: a meta-analysis. Support Care Cancer. 2020;28:27012712.

8. DE Felice F, Pranno N, Papi P, Brugnoletti O, Tombolini V, Polimeni A. Xerostomia and clinical outcomes in definitive intensity modulated radiotherapy (IMRT) versus threedimensional conformal radiotherapy (3D-CRT) for head and neck squamous cell carcinoma: a meta-analysis. In Vivo. 2020;34:623-629.

9. Otto K. Volumetric modulated arc therapy: IMRT in a single gantry arc. Med Phys. 2008;35:310-317.

10. Holt A, Van Gestel D, Arends MP, Korevaar EW, Schuring D, Kunze-Busch MC, et al. Multi-institutional comparison of volumetric modulated arc therapy vs. intensity-modulated radiation therapy for head-and-neck cancer: a planning study. Radiat Oncol. 2013;8:26.

11. Studenski MT, Bar-Ad V, Siglin J, Cognetti D, Curry J, Tuluc $\mathrm{M}$, et al. Clinical experience transitioning from IMRT to VMAT for head and neck cancer. Med Dosim. 2013;38:171175.

12. Verbakel WF, Cuijpers JP, Hoffmans D, Bieker M, Slotman BJ, Senan S. Volumetric intensity-modulated arc therapy vs. conventional IMRT in head-and-neck cancer: a comparative planning and dosimetric study. Int J Radiat Oncol Biol Phys. 2009;74:252-259.

13. Kumar L, Yadav G, Raman K, Bhushan M, Pal M. The dosimetric impact of different photon beam energy on RapidArc radiotherapy planning for cervix carcinoma. J Med Phys. 2015;40:207-213.
14. Kumar L, Yadav G, Samuvel KR, Bhushan M, Kumar P, Suhail M, et al. Dosimetric influence of filtered and flattening filter free photon beam on rapid arc (RA) radiotherapy planning in case of cervix carcinoma. Rep Pract Oncol Radiother. 2017;22:10-18.

15. Diallo I, Haddy N, Adjadj E, Samand A, Quiniou E, Chavaudra J, et al. Frequency distribution of second solid cancer locations in relation to the irradiated volume among 115 patients treated for childhood cancer. Int J Radiat Oncol Biol Phys. 2009;74:876-883.

16. Nagarajan M, Banu R, Sathya B, Sundaram T, Chellapandian TP. Dosimetric evaluation and comparison between volumetric modulated arc therapy (VMAT) and intensity modulated radiation therapy (IMRT) plan in head and neck cancers. Gulf J Oncolog. 2020;1:45-50.

17. Osborn J. Is VMAT beneficial for patients undergoing radiotherapy to the head and neck? Radiography (Lond). 2017;23:73-76.

18. ICRU Report 83. Prescribing, recording, and reporting intensity-modulated photon-beam therapy (IMRT). ICRU Report. Bethesda: ICRU. 2010; 83.

19. IMPAC Medical Systems, Inc. Monaco ${ }^{\circledR}$ training guide. Stockholm: Elekta AB; 2013.

20. Kumar L, Yadav G, Kishore V, Bhushan M, Gairola M, Tripathi D. Validation of the RapidArc delivery system using a volumetric phantom as per task group report 119 of the American Association of Physicists in Medicine. J Med Phys. 2019;44:126-134.

21. Radhakrishnan S, Chandrasekaran A, Sarma Y, Balakrishnan S, Nandigam J. Dosimetric comparison between single and dual arc-volumetric modulated arc radiotherapy and intensity modulated radiotherapy for nasopharyngeal carcinoma using a simultaneous integrated boost technique. Asian Pac J Cancer Prev. 2017;18:1395-1402.

22. Nithya L, Raj NA, Kumar A, Rathinamuthu S, Pandey MB. Comparative analysis of volumetric-modulated arc therapy and intensity-modulated radiotherapy for base of tongue cancer. J Med Phys. 2014;39:121-126.

23. Kumar L, Bhushan M, Kishore V, Yadav G, Gurjar OP. Dosimetric validation of Acuros ${ }^{\circledR} \mathrm{XB}$ algorithm for RapidArc ${ }^{\mathrm{nt}}$ treatment technique: a post software upgrade analysis. J Cancer Res Ther. 2021. doi: 10.4103/jcrt.JCRT_1154_19.

24. Stovall M, Blackwell CR, Cundiff J, Novack DH, Palta JR, 
Wagner LK, et al. Fetal dose from radiotherapy with photon beams: report of AAPM Radiation Therapy Committee Task Group No. 36. Med Phys. 1995;22:63-82.

25. Edwards CR, Mountford PJ. Near surface photon energy spectra outside a 6 MV field edge. Phys Med Biol. 2004;49: N293-N301.

26. Gayen S, Kombathula SH, Manna S, Varshney S, Pareek P. Dosimetric comparison of coplanar and non-coplanar volumetric-modulated arc therapy in head and neck cancer treated with radiotherapy. Radiat Oncol J. 2020;38:138147.

27. Hall EJ. Intensity-modulated radiation therapy, protons, and the risk of second cancers. Int J Radiat Oncol Biol Phys. 2006;65:1-7.
28. Hall EJ, Wuu CS. Radiation-induced second cancers: the impact of 3D-CRT and IMRT. Int J Radiat Oncol Biol Phys. 2003;56:83-88.

29. Followill D, Geis P, Boyer A. Estimates of whole-body dose equivalent produced by beam intensity modulated conformal therapy. Int J Radiat Oncol Biol Phys. 1997;38:667-672.

30. Hauri P, Schneider U. Whole-body dose equivalent including neutrons is similar for $6 \mathrm{MV}$ and $15 \mathrm{MV}$ IMRT, VMAT, and 3D conformal radiotherapy. J Appl Clin Med Phys. 2019;20:56-70.

31. Kry SF, Bednarz B, Howell RM, Dauer L, Followill D, Klein E, et al. AAPM TG 158: Measurement and calculation of doses outside the treated volume from external-beam radiation therapy. Med Phys. 2017;44:e391-e429. 